\title{
Variables motivacionales implicadas en la actividad física en el alumnado de
}

\section{secundaria}

\author{
Antonio López Castedo, Mª Esther López Pérez \\ Facultad de Ciencias de la Educación (Campus de Ourense). Universidad de Vigo
}

\begin{abstract}
Resumen
El objetivo de esta investigación es conocer las variables motivacionales implicadas en la actividad física en función del sexo y rendimiento académico de 326 adolescentes. Se aplicó el Autoinforme de Motivos para la Práctica de Ejercicio Físico (AMPEF) en versión española de Capdevila, Niñarola y Pintanel (2004). Se aprecian diferencias significativas, según el sexo, en dimensiones de Peso e Imagen Corporal, Diversión y Bienestar, Prevención de Salud Positiva, Competición, Afiliación, Fuerza y Resistencia muscular, Reconocimiento social y Desafío; mientras que, en rendimiento académico, se manifiestan en Diversión y Bienestar, Prevención y Salud Positiva, Afiliación, Fuerza y Resistencia Muscular y Desafío.

Palabras clave: motivación, actividad física, adolescencia, educación.
\end{abstract}

\section{Introducción}

En la actualidad, existe una preocupación generalizada en cuanto a la práctica de actividad física de forma regular. Este sentimiento está presente en todas las etapas de la vida, pero preferentemente en la adolescencia. Por una parte, por ser un momento de grandes cambios morfológicos y hormonales, en el que se produce una restructuración de la imagen de uno mismo y un cambio en el pensamiento, lo que hace que sea un lugar idóneo para adquirir unos comportamientos adecuados que mejoren la calidad de vida de los estudiantes. Por otra, son numerosos los estudios que muestran un importante descenso en la motivación e interés hacia la actividad física en los adolescentes (Chillón et al., 2009; Macarro, Romeo y Torres (2010; Riewald, 2003; Wall y Cot, 2007), y un incremento del sedentarismo (Cecchini, Méndez y Contreras, 2005; García Ferrando y Lopis, 2011), lo que puede llevar a una asimilación de formas de vida que alteren su bienestar.

Los avances en este campo se encaminan en la elaboración de directrices básicas para el desarrollo de la actividad físico-deportiva (Sierra, 2005), y sobre todo, hacia la promoción de salud, de bienestar y de calidad de vida (Allsen, Harrison y Vance, 2001; Reinboth y Duda, 2006). Además, son numerosos los estudios que relacionan la práctica regular de la actividad física con los factores motivacionales (Barbosa, 2005; Capozzoli, 2006).

Viendo el estado de la cuestión, rápidamente se observa que esta tendencia al abandono y a la desmotivación preocupa bastante a los investigadores (Cervelló, 1996; Corbin, 2002; Fairclough, 2003; Ntoumanis, Taylor y Standage 2010; Papaioannou, 1997; Standage y Gillison, 2007). Por este motivo, en las últimas décadas han aparecido diversos estudios que tratan de exponer numerosos motivos tanto para la práctica como para el abandono, en los que se observa que el constructo de la motivación y los factores que influyen sobre ella como la autoestima, la competencia o incompetencia percibida, la autonomía o las relaciones sociales con el entorno, son estudiados bajo el abrigo de teorías motivacionales entre las que actualmente destaca la teoría de la autodeterminación (Biddle y Wang, 2003; Ntoumanis, 2001; Ntoumanis y Standage, 2009; Ntoumanis, Taylor y Standage, 2010; Vansteenkiste Lens y Deci, 2006).

Es un reto enorme, sobre todo por las características generales del estadio adolescente, la apuesta por un estudio motivacional en el alumnado de secundaria hacia la actividad física. Así pues, es esencial tener presentes las características generales del período adolescente, ya que es una fase de cambio y de tránsito donde el desarrollo se ve afectado, y esa metamorfosis es la base de la adolescencia. Por tanto, nuestro objetivo es profundizar en las variables motivacionales implicadas en la actividad física atendiendo al sexo y rendimiento académico del alumnado de secundaria.

Método
Participantes
La población objeto de estudio se corresponde con los
alumnos/as de Educación Secundaria Obligatoria de la
provincia de Ourense, con edades comprendidas entre
los 12 y 16 años. Se seleccionó una muestra de un total
de 326 alumnos/as, de centros públicos y privados-
concertados y que voluntariamente accedieron a
colaborar en esta investigación. La caracterización de la
muestra se distribuye de la siguiente forma: las mujeres
representan el 58.8\% (192) y los hombres el $41.2 \%$
(134) del total. En cuanto a la edad, entre 13 y 15 años
se aglutinan el $71.5 \%$ de la muestra, siendo los grupos
más reducidos los de 16 y 12 años. La edad media es de


13.86 años, con una desviación típica de 1.25. Con relación al tipo de centro en el que estudian, se observa un porcentaje ligeramente superior en los centros públicos $(57.1 \%$ frente a los privados-concertados (42.9\%). Respecto al curso, se constata que el grupo más numeroso es el formado por $3^{\circ}$ de la ESO con un $27.3 \%$ (89), mientras que, en el resto de cursos su distribución es bastante homogénea $\left(1^{\circ}\right.$ y $4^{\circ}=24.5 \%$; $2^{\circ}$ $=23.6 \%$ ). En el rendimiento académico, la mayoría aprueba todas las asignaturas (72.7\%), otros pasan de curso pero con alguna materia suspensa (12.9\%) y los que han repetido curso alguna vez representan el 14.4\%. Finalmente, atendiendo al tipo de familia, el $72.4 \%$ engloba a familias estructuradas, mientras que el $27.6 \%$ a familias desestructuradas.

\section{Instrumentos}

En primer lugar, se utilizó un Cuestionario estructurado "ad hoc" relativo a las variables sociodemográficas entre las que se incluyen edad, sexo, tipo de centro, curso, rendimiento académico y tipo de familia. En segundo lugar, el Autoinforme de Motivos para la Práctica de Ejercicio Físico (AMPEF), que es la traducción del Exercise Motivations Inventory (EMI2) de Markland e Ingledew (1997) y en versión española de Capdevila, Niñarola y Pintanel (2004). Se trata de un cuestionario formado por 48 ítems con un formato tipo Likert de 0 ("nada verdadero para mí") a 10 ("totalmente verdadero para mí) en función del grado de acuerdo que refleje su motivación para hacer ejercicio físico. Su objetivo es estudiar la motivación y la adherencia hacia el ejercicio físico en relación a un estilo de vida saludable global. Se centra en evaluar los diferentes motivos que influyen en la decisión de una persona para iniciar un estilo de vida más activo. Está formado por 11 dimensiones que se refieren a:

\begin{tabular}{|c|c|c|}
\hline Dimensión & Interpretación & Indicador \\
\hline $\begin{array}{l}\text { Peso e Imagen } \\
\text { Corporal }\end{array}$ & $\begin{array}{l}\text { Indica que los motivos } \\
\text { determinantes para realizar } \\
\text { ejercicio son el control del } \\
\text { peso y el deseo de tener un } \\
\text { cuerpo que guste a los demás. }\end{array}$ & $\begin{array}{l}\text { Ítems: } 27, \\
13,40,1 \text {, } \\
29,41,16 .\end{array}$ \\
\hline $\begin{array}{l}\text { Diversión } \quad \text { y } \\
\text { Bienestar }\end{array}$ & $\begin{array}{l}\text { El objetivo de la práctica de } \\
\text { ejercicio físico es obtener } \\
\text { sensaciones relacionadas con } \\
\text { el bienestar, la diversión, la } \\
\text { autosatisfacción, la carga de } \\
\text { energías o el sentirse bien. }\end{array}$ & $\begin{array}{c}\text { Ítems: 45, } \\
\text { 7, 3, 21, } \\
34 .\end{array}$ \\
\hline $\begin{array}{l}\text { Prevención y } \\
\text { Salud Positiva }\end{array}$ & $\begin{array}{l}\text { El ejercicio físico se practica } \\
\text { para evitar posibles } \\
\text { problemas de salud y para } \\
\text { mantener o mejorar la salud } \\
\text { actual. }\end{array}$ & $\begin{array}{c}\text { Ítems: 2, } \\
\text { 19, 5, 32, } \\
14 .\end{array}$ \\
\hline
\end{tabular}

\begin{tabular}{|c|c|c|}
\hline Competición & $\begin{array}{l}\text { La importancia de comparar } \\
\text { las propias habilidades } \\
\text { físicas o deportivas con } \\
\text { terceras personas, y la } \\
\text { sensación placentera de la } \\
\text { victoria frente a los rivales en } \\
\text { actividades deportivas. }\end{array}$ & $\begin{array}{l}\text { Ítems: 24, } \\
37,47,10 .\end{array}$ \\
\hline Afiliación & $\begin{array}{l}\text { Motivos de tipo social para } \\
\text { realizar ejercicio físico, como } \\
\text { son el sentirse miembro de un } \\
\text { grupo, club o servicio } \\
\text { deportivo. }\end{array}$ & $\begin{array}{c}\text { Ítems: 8, } \\
\text { 35, 46, } 22 .\end{array}$ \\
\hline $\begin{array}{l}\text { Fuerza } \\
\text { Resistencia } \\
\text { Muscular }\end{array}$ & $\begin{array}{l}\text { Se practica ejercicio físico } \\
\text { para mejorar específicamente } \\
\text { el componente muscular de la } \\
\text { condición física. }\end{array}$ & $\begin{array}{c}\text { Ítems: 6, } \\
\text { 33, 44, } 20 .\end{array}$ \\
\hline $\begin{array}{l}\text { Reconocimien } \\
\text { to Social }\end{array}$ & $\begin{array}{l}\text { Los motivos están } \\
\text { relacionados con la búsqueda } \\
\text { de alabanzas por parte de los } \\
\text { compañeros y amigos. }\end{array}$ & $\begin{array}{l}\text { Ítems: } \\
4,42,17 \text {, } \\
30 .\end{array}$ \\
\hline $\begin{array}{l}\text { Control del } \\
\text { Estrés }\end{array}$ & $\begin{array}{l}\text { Se utiliza la práctica de } \\
\text { ejercicio físico como una } \\
\text { estrategia de afrontamiento } \\
\text { de la ansiedad y del estrés. }\end{array}$ & $\begin{array}{c}\text { Ítems: } 31, \\
43,18 .\end{array}$ \\
\hline $\begin{array}{l}\text { Agilidad } \quad \mathrm{y} \\
\text { Flexibilidad }\end{array}$ & $\begin{array}{l}\text { Relacionados con el } \\
\text { componente de flexibilidad de } \\
\text { la condición física saludable, } \\
\text { así como con la mejora o el } \\
\text { mantenimiento de la agilidad } \\
\text { corporal. }\end{array}$ & $\begin{array}{c}\text { Ítems: 25, } \\
\text { 38, } 11 .\end{array}$ \\
\hline Desafío & $\begin{array}{l}\text { Se entiende como una } \\
\text { herramienta para plantearse } \\
\text { objetivos a corto plazo, de } \\
\text { manera que el mantenimiento } \\
\text { de un estilo de vida activo se } \\
\text { convierte en un reto a } \\
\text { superar. }\end{array}$ & $\begin{array}{l}\text { Ítems: 12, } \\
\text { 26, 48, } 39 .\end{array}$ \\
\hline $\begin{array}{l}\text { Urgencias de } \\
\text { Salud }\end{array}$ & $\begin{array}{l}\text { Necesidad de practicar } \\
\text { ejercicio físico por pres- } \\
\text { cripción médica, habitual- } \\
\text { mente como tratamiento o por } \\
\text { un trastorno de salud } \\
\text { diagnosticado. }\end{array}$ & $\begin{array}{c}\text { Ítems: } 36, \\
23,9 .\end{array}$ \\
\hline
\end{tabular}

El estudio de fiabilidad ha mostrado una correlación significativa entre test y retest después del transcurso de doce meses $(r=.85)$ lo que indica que se mantiene a lo largo del tiempo el mismo nivel general de motivación. La consistencia interna (Alfa de Cronbach) para el total de la escala es de .92 y para las distintas dimensiones oscila entre .71 y .92 .

\section{Procedimiento}

Previamente a la recogida de dato, se procedió a contactar con el equipo directivo y los orientadores de los centros, con el objeto de explicarles la finalidad y alcance de la investigación y proponerles su participación voluntaria. Obtenido el consentimiento de la dirección de los centros, se mantiene una reunión informativa para dar a conocer el estudio y que éstos lo trasladen a toda la comunidad educativa. Los tutores participan de forma voluntaria $\mathrm{y}$ no remunerada. Paralelamente, se informa a los padres sobre la investigación y se obtiene el consentimiento de la participación de sus hijos en el estudio. A los 
adolescentes que deciden colaborar se les pide que sean lo más sinceros posible a la hora de contestar y que no dejen ninguna pregunta sin responder. Las instrucciones explicativas son las mismas en todas las aulas y el mismo encuestador, a fin de evitar un factor de sesgo. Es necesario señalar la receptividad de todos los profesores, alumnos y padres a los que se les solicitó colaboración, así como la alta participación de los estudiantes en esta investigación.

\section{Resultados}

En nuestra investigación la fiabilidad obtenida a través del Alfa de Cronbach ha sido para el total de .95 y para las distintas dimensiones entre .75 y .94. Por tanto, consideramos que este estudio alberga unos índices de fiabilidad muy satisfactorios.

Por lo que respecta a las diferentes dimensiones del Cuestionario de Motivación (AMPEF) en función del sexo (tabla 1), se pone de manifiesto que en el Peso e Imagen Corporal, Prevención de Salud Positiva y Control del Estrés, la media más alta corresponde a las mujeres en relación a los hombres. Por otra parte, en las dimensiones de Diversión y Bienestar, Competición, Afiliación, Fuerza y Resistencia Muscular, Reconocimiento Social, Agilidad y Flexibilidad, Desafío y Urgencia de Salud, se observa que la media mayor corresponde a los hombres en relación a las mujeres.

Tabla 1

Medias, desviaciones típicas y prueba “ $t$ " de las dimensiones del AMPEF en función del Sexo

\begin{tabular}{cclc}
\hline Dimensión & Sexo & \multicolumn{1}{c}{$\overline{\boldsymbol{Z}}(5 x)$} & $\mathrm{t}(\mathrm{p})$ \\
\hline Peso e Imagen & Mujer & $43.90(16.60)$ & 4.478 \\
Corporal & Hombre & $40.00(15.99)$ & $(<.035)$ \\
Diversión y & Mujer & $33.53(11.81)$ & 7.058 \\
Bienestar & Hombre & $39.96(10.70)$ & $(<.008)$ \\
Prevención y & Mujer & $34.36(8.81)$ & 3.382 \\
Salud Positiva & Hombre & $32.48(9.48)$ & $(<.050)$ \\
Competición & Mujer & $17.53(10.01)$ & 30.646 \\
& Hombre & $23.56(9.18)$ & $(<.000)$ \\
Afiliación & Mujer & $23.45(10.13)$ & 9.123 \\
& Hombre & $26.89(10.02)$ & $(<.003)$ \\
Fuerza y Resis- & Mujer & $25.42(9.19)$ & 25.122 \\
tencia Muscular & Hombre & $30.22(7.45)$ & $(<.000)$ \\
Reconocimiento & Mujer & $18.97(9.61)$ & 3.519 \\
Social & Hombre & $21.13(10.98)$ & $(<.050)$ \\
Control del & Mujer & $14.92(9.05)$ & .305 \\
Estrés & Hombre & $14.36(8.91)$ & $(.581)$ \\
Agilidad y & Mujer & $17.03(7.71)$ & .167 \\
Flexibilidad & Hombre & $17.39(7.78)$ & $(.683)$ \\
Desafío & Mujer & $25.64(9.92)$ & 6.626 \\
& Hombre & $28.49(9.77)$ & $(<.010)$ \\
Urgencias de & Mujer & $10.11(7.28)$ & 4.382 \\
Salud & Hombre & $11.82(7.18)$ & $(<.037)$ \\
\hline
\end{tabular}

La prueba " $\mathrm{t}$ ” de muestras independientes, nos indica que existen diferencias significativas en Peso e Imagen Corporal $(\mathrm{t}=4.478 ; p<.035)$, Diversión y Bienestar $(\mathrm{t}=$ 7.058; $p<.008)$, Prevención de Salud Positiva $(\mathrm{t}=$ 3.382; $p<.050)$, Competición ( $\mathrm{t}=30.646 ; p<.000)$,
Afiliación ( $\mathrm{t}=9.123 ; p<.003)$, Fuerza y resistencia muscular $(\mathrm{t}=25.122 ; p<.000)$, Reconocimiento social $(\mathrm{t}$ $=3.519 ; p<.050)$ y Desafío $(\mathrm{t}=6.626 ; p<.010)$. Dado que los resultados de la prueba " $\mathrm{t}$ " muestran diferencias significativas $(\mathrm{p}<.05)$ en Peso e Imagen Corporal y Prevención de Salud Positiva, éstas se ponen de manifiesto con una media más alta de las mujeres en relación a los hombres, por el contrario, en las dimensiones Diversión y Bienestar, Competición, Afiliación, Fuerza y resistencia muscular, Reconocimiento social y Desafío, los hombres manifiestan una media más alta que las mujeres.

En cuanto a las distintas dimensiones del Cuestionario de Motivación (AMPEF) en función del rendimiento académico (tabla 2), podemos observar que en el Peso e Imagen Corporal, Prevención de Salud Positiva, Competición, Afiliación, Fuerza y Resistencia Muscular y Agilidad y flexibilidad, las medias más altas corresponden a los alumnos que aprueban todo, mientras que las más bajas se muestran en los que repiten curso.

Tabla 2

Medias, desviaciones típicas y Anovas de las dimensiones del AMPEF en función del Rendimiento Académico

\begin{tabular}{|c|c|c|c|}
\hline Dimensión & Rendimiento & $\bar{X}(S x)$ & $\mathrm{F}(p)$ \\
\hline Peso e Imagen & Aprueba todo & 43.43(15.70) & 2.126 \\
\hline \multirow[t]{2}{*}{ Corporal } & No repite & 39.67(18.19) & \multirow[t]{2}{*}{$(.121)$} \\
\hline & Repite & 38.89(18.14) & \\
\hline \multirow{3}{*}{$\begin{array}{l}\text { Diversión y } \\
\text { Bienestar }\end{array}$} & Aprueba todo & 36.29(10.98) & \multirow{3}{*}{$\begin{array}{c}7.050 \\
(<.001)\end{array}$} \\
\hline & No repite & 29.81(11.41) & \\
\hline & Repite & 32.62 12.50) & \\
\hline Prevención y & Aprueba todo & 35.05 (8.60) & \multirow{3}{*}{$\begin{array}{l}12.204 \\
(<.000)\end{array}$} \\
\hline \multirow[t]{2}{*}{ Salud Positiva } & No repite & 30.26 (8.67) & \\
\hline & Repite & 29.15(10.15) & \\
\hline \multirow[t]{3}{*}{ Competición } & Aprueba todo & 20.59(10.05) & \multirow{3}{*}{$\begin{array}{l}1.585 \\
(.206)\end{array}$} \\
\hline & No repite & $19.12(9.40)$ & \\
\hline & Repite & 17.89(10.83) & \\
\hline \multirow[t]{3}{*}{ Afiliación } & Aprueba todo & $26.00(9.97)$ & \multirow{3}{*}{$\begin{array}{c}5.607 \\
(<.004)\end{array}$} \\
\hline & No repite & 22.21 (9.76) & \\
\hline & Repite & 21.49(10.81) & \\
\hline \multirow{3}{*}{$\begin{array}{c}\text { Fuerza y } \\
\text { Resistencia } \\
\text { Muscular }\end{array}$} & Aprueba todo & 28.14 (8.79) & \multirow{3}{*}{$\begin{array}{c}3.316 \\
(<.038)\end{array}$} \\
\hline & No repite & $25.98(8.76)$ & \\
\hline & Repite & $24.89(8.67)$ & \\
\hline \multirow{3}{*}{$\begin{array}{l}\text { Reconocim. } \\
\text { Social }\end{array}$} & Aprueba todo & $20.00(10.24)$ & \multirow{3}{*}{$\begin{array}{c}.108 \\
(.898)\end{array}$} \\
\hline & No repite & 19.24(10.71) & \\
\hline & Repite & 19.68 (9.99) & \\
\hline \multirow{3}{*}{$\begin{array}{l}\text { Control del } \\
\text { Estrés }\end{array}$} & Aprueba todo & $14.78(9.21)$ & \multirow{3}{*}{$\begin{array}{l}.318 \\
(.728)\end{array}$} \\
\hline & No repite & 13.69 (8.65) & \\
\hline & Repite & 15.09 (8.14) & \\
\hline Agilidad y & Aprueba todo & $17.51(7.76)$ & \multirow{3}{*}{$\begin{array}{l}.842 \\
(.432)\end{array}$} \\
\hline \multirow[t]{2}{*}{ Flexibilidad } & No repite & $16.41(7.67)$ & \\
\hline & Repite & $16.15(7.60)$ & \\
\hline \multirow[t]{3}{*}{ Desafío } & Aprueba todo & $27.70(9.79)$ & \multirow{3}{*}{$\begin{array}{c}3.672 \\
(<.026)\end{array}$} \\
\hline & No repite & 23.81(10.32) & \\
\hline & Repite & 25.02 (9.97) & \\
\hline \multirow{3}{*}{$\begin{array}{l}\text { Urgencias de } \\
\text { Salud }\end{array}$} & Aprueba todo & $10.89(7.04)$ & \multirow{3}{*}{$\begin{array}{c}.537 \\
(.585)\end{array}$} \\
\hline & No repite & 11.43 (7.61) & \\
\hline & Repite & $9.89(8.21)$ & \\
\hline
\end{tabular}

Por otra parte, en las dimensiones de Diversión y Bienestar, Reconocimiento Social y Desafío, los que aprueban todo registran la media más alta, por el 
contrario, la más baja se sitúa en los alumnos que repiten; en Control del Estrés, los alumnos que repiten presentan la media más alta, mientras que los que no repiten la más baja, y, por último, en la dimensión Urgencia de Salud, los sujetos que no repiten registran la media más alta, a la vez que en los que repiten, se encuentra la más baja.

El análisis inferencial (ANOVA) nos pone de manifiesto que Diversión y Bienestar $(\mathrm{F}=7.050$; $p<.001)$, Prevención y Salud Positiva $(\mathrm{F}=12.204$; $p<.000)$, Afiliación $(\mathrm{F}=5.607 ; p<.004)$, Fuerza $\mathrm{y}$ Resistencia Muscular ( $\mathrm{F}=3.316 ; p<.038)$, y Desafío ( $\mathrm{F}$ $=3.672 ; \quad p<.026)$, son fuentes significativas de variación; no encontrando diferencias significativas en las otras dimensiones.

Los resultados de las pruebas a posteriori (Scheffé) muestran diferencias significativas $(p<.05)$ en la dimensión de Diversión y bienestar, entre los que aprueban todo y los que no repiten; en Prevención y salud positiva, se encuentran entre los que aprueban todo y los que no repiten, y entre los que aprueban todo y los que han repetido; en Afiliación, se observan entre los que aprueban todo y los que no repiten, y los que aprueban todo y los que han repetido; en la dimensión de Fuerza y resistencia muscular, se presentan entre los aprueban todo y los que no repite, y entre los que aprueban todo y los que han repetido; y, finalmente, en Desafío, se dan entre los que aprueban todo y los que no repiten, y los que aprueban todo y los que han repetido.

\section{Discusión}

La consistencia interna de la escala y de las dimensiones se ha determinado a partir del cálculo del coeficiente alfa de Cronbach, siendo altamente satisfactoria y en consonancia con los resultados de Markland y Ingledew (1997) y la versión española de Capdevila, Niñarola y Pintanel (2004).

En general, los adolescentes dan mayor trascendencia apariencia, aunque también existen otros motivos, como la diversión, la relación social con otros iguales o el bienestar (Castillo y Balaguer, 2001; Piéron et al., 1999; Ponseti, Gili, Palou, y Borrás, 1998; Yan y McCullagh, 2004). Esto sugiere la importancia de la preocupación por la imagen corporal típica del adolescente, como motor para la participación en actividades físicas, así como la búsqueda de la propia identidad y la necesidad de interaccionar y relacionarse con otros. La orientación a metas (Guillén y Álvarez-Malé, 2010) y la orientación al ego (Jeffrey, 2013) adquieren relevancia y pueden influir en la toma de decisiones con respecto a la participación en actividades físicas recreativas o competitivas (Medic, Young y Grove, 2013).

Por otra parte, mujeres y hombres se comportan de manera distinta en cuanto a motivos para realizar actividad física. Los motivos de las mujeres se relacionan con el cuidado de la imagen y el control del peso corporal, para prevenir problemas de salud y mantener/mejorar la salud actual (Pavón y Moreno, 2008; Pinto, Santos y Legani, 2012). Por su parte, los hombres presentan entre sus principales motivos la competición, rendimiento físico, reconocimiento social, diversión/bienestar y desafío (Águila, Sicilia, Muyor y Orta, 2009; Pavón y Moreno, 2008; Pinto, Santos y Legani, 2012).

El factor control del estrés resultó más relevante para las mujeres y aquellos adolescentes que repiten curso. Las demandas psicosociales y los cambios fisiológicos podrían asociarse con estos hallazgos. Para los adultos jóvenes, las exigencias de cumplir con diversos roles pueden resultar en niveles de estrés que requieran de estrategias como la actividad física (Ingledew, Markland y Ferguson, 2009; Jiménez-Torres, GodoyIzquierdo y Godoy, 2012). Asimismo, el manejo del estrés es un aspecto relevante para el género femenino en general (Jeffrey, 2013).

Cuando se relacionan los motivos con el rendimiento académico, se ha encontrado que los adolescentes que aprueban todo llevan a cabo una actividad física por motivos de diversión/bienestar, afiliación, fuerza y resistencia muscular y desafío. Distintos estudios señalan que los jóvenes tienden que a mostrar un rendimiento académico óptimo, niveles más altos de concentración, y un mejor comportamiento que incide sobre los procesos de aprendizaje (Tremblay, Inman y Willms, 2000). Por tanto, es fundamental conocer el proceso motivacional de los adolescentes teniendo en cuenta tres elementos esenciales: la motivación del alumno hacia la actividad física, las diferencias motivacionales según las distintas etapas escolares y, diferente motivación y rendimiento académico (Flores y Gómez, 2010).

Por último, el conocimiento generado en esta investigación posibilita una comprensión y un acercamiento más profundo de las variables motivacionales en el alumnado de secundaria, aspectos que favorecerán la toma de decisiones futuras en materia de planificación, organización, promoción e intervención para impulsar la actividad física como una actividad que produce incuestionables beneficios en cuanto a salud y calidad de vida.

\section{Bibliografía}

Águila, C., Sicilia, A., Muyor, J., y Orta, A. (2009). Cultura posmoderna y perfiles de práctica en los centros deportivos municipales. Revista Internacional de Medicina y Ciencias de la Actividad Física y el Deporte, 9(33), 81-95.

Allsen, P.E., Harrison, J.M., y Vance, B. (2001). Exercício e qualidade de vida:Uma abordagem personalizada. São Paulo, Brasil: Manole.

Barbosa, M. L.L. (2005). Propiedades métricas do inventario de Motivaçao à práctica regular de actividade física (IMPRAF-126). Porto Alegre: Escola de Educaçao Física.

Biddle, S. J., y Wang, C. K. (2003). Motivation and self-perception profiles and links with physical activity in adolescent girls. Journal of Adolescence, 26, 687-701.

Capdevila, L., Niñerola, J. y Pintanel, M. (2004). Motivación y actividad física: El autoinforme de motivos para la práctica de ejercicio físico (AMPEF). Revista de Psicología del Deporte, 13(1), 55-74. 
Capozzoli, C. J., (2006). Motivaçao à práctica regular de actividades físicas: um estudo com practicantes em academias de ginásctica de Porto Alegre. Porto Alegre: Escola de Educaçao Física.

Castillo, I., y Balaguer, I. (2001). Dimensiones de los motivos de práctica deportiva de los adolescentes valencianos escolarizados. Apunts: Educación Física y Deportes, 63, 22-29.

Cechini, J. A., Méndez, A., y Contreras, O. R. (2005).Motivos de abandono de la práctica del deporte juvenil. Castilla-La Mancha: Ediciones de la universidad de Castilla-La Mancha.

Cervelló, E. (1996). La motivación y el abandono deportivo desde la perpectiva de las metas de logro. Valencia: Servicio de Publicaciones de la Universidad de Valencia.

Chillón, P., Ortega, F., Ruiz, J., Pérez, I., MartínMatillas, M., Valtueña, J., Gómez- Martínez, .... y Delgado, M. (2009). Socio-economic factors and active commuting to school in urban Spanish adolescents: the AVENA study. European Journal of PublicHealth, 19 (5), 470-476.

Corbin, C.B. (2002). Physical education as an agent of change. Quest 54, 182-95.

Fairclough, S.J. (2003). Girls' physical activity during high school physical education: influences of body composition and cardiorespiratory fitness. Journal of Teaching in Physical Education 22, 382-95.

Flores, R., y Gómez, J. (2010). Un estudio sobre la motivación hacia la escuela secundaria en estudiantes mexicanos. Revista Electrónica de Investigación Educativa, 12(1), 1-21.

García-Ferrando, M., y Llopis, R. (2011). Ideal democrático y bienestar personal. Encuesta sobre hábitos deportivos en España 2010. Madrid. Ministerio de Cultura/Consejo Superior de Deportes.

Guillén, F., y Álvarez-Malé, M. L. (2010). Relación entre los motivos de la práctica deportiva y la ansiedad en jóvenes nadadores de competición. Revista Iberoamericana de Psicología del Ejercicio y el Deporte, 5(2), 233-252.

Ingledew, D. K., Markland, D., y Ferguson, E. (2009). Three levels of exercise motivation. Applied Psychology: Health and Well-being, 1(3), 336-355.

Jeffrey, P. (2013). Physical activity behaviors, motivation, and self-efficacy among college students. College Student Journal, 47(1), 64-74.

Jiménez-Torres, M. G., Godoy-Izquierdo, D., y Godoy, G. J. (2012). Relación entre los motivos para la práctica físico-deportiva y las experiencias de flujo en jóvenes: diferencias en función del sexo. Universitas Psychologica, 11(3), 909-920.

Macarro, J., Romero, C., y Torres, J. (2010). Motivos de abandono de la práctica de actividad físico-deportiva en los estudiantes de Bachillerato de la provincia de Granada. Revista de Educación, 343, 495-519.

Markland, D., y Ingledew, D. K. (1997). The measurement of exercise mo-tives: Factorial validity and invariance across gender of a revised Exer-cise Motivations Inventory. British Journal of Health Psychology, 2, 361-376.
Medic, N., Young, B. W., y Grove, J. R. (2013). Perceptions of five-year competitive categories: model of how relative age influences competitiveness in masters sport. Journal of Sports Science \& Medicine, 12(4), 724-729.

Ntoumanis, N. (2001). A self-determination approach to the understanding of motivation in physical education. British Journal of Educational Psychology, 71, 225242.

Ntoumanis, N., y Standage, M. (2009). Motivation in physical education classes: A self-determination theory perspective. Theory and Research in Education, 7, 194-202.

Ntoumanis, N; Taylor, I.M., y Standage, M. (2010). Testing a model of antecedents and consequences of defensive pessimism and self-handicapping in school physical education. Journal of Sports Sciences, 28(14), 1515-1525.

Papaioannou, A. (1997). Perceptions of motivational climate, perceived competence, and motivation of students of varying age and sport experience. Perceptual and Motor Skills, 85, 419-430.

Pavón, A., y Moreno, J. (2008). Actitud de los universitarios ante la práctica física deportiva: diferencias por géneros. Revista de Psicología del Deporte, 17(1), 723.

Piéron, M., Telama, R., Almond, L., y Carreiro da Costa, F. (1999). Estilo de vida de jóvenes europeos: Un estudio comparativo. Revista de Educación Física, 76, 5-13.

Pinto, D., Santos, R. y Legani, E. (2012). Motivos para la práctica de ejercicio físico en universitarios de acuerdo con el índice masa corporal. Revista Brasileira de Atividade Física \& Saúde, 17(4), 270274.

Ponseti, F., Gili, M., Palou, P., y Borrás, P. (1998). Intereses, motivos y actitudes hacia el deporte en adolescentes: diferencias en función del nivel de práctica. Revista de Psicología del Deporte, 7(2), 259274.

Reinboth, M., y Duda, J.L. (2006). Perceived motivational climate, need satisfaction and indices of well-being in a team sports: A longitudinal perspective. Psychology of Sport and Exercise, 7, 269-286.

Riewald, S.T. (2003). Strategies to prevent dropout from youth athletics. New Studies in Athletics, 18(3), 21-26.

Sierra, A. (2005). Formación docente para el control de la carga en la clase de Educación Física. Revista de Investigación en Educación, 2, 33-48.

Standage, M., y Gillison, F. (2007). Students' motivational responses toward school physical education and their relationship to general self-esteem and health-related quality of life. Psychology of Sport and Exercise, 8, 704-721.

Tremblay, M., Inman, J., y Willms, J. (2000). The Relationship Between Physical Activity, SelfEsteem, and Academic Achievement in 12-Year-Old Children. Pediatric Exercise Science, 12, 312-324.

Vansteenkiste, M., Lens, W., y Deci, E.L. (2006). Intrinsic Versus Extrinsic Goal Contents in Self- 
Determination Theory: Another Look at the Quality of Academic Motivation. Educational Psychologist, 41(1), 19-31.

Wall, M., y Cot, J. (2007). Developmental activities that lead to dropout and investment in sport.Physical Education and Sport Pedagogy, 12, 77-88.
Yan, J. H., y McCullagh, P. J. (2004). Cultural influence on youth's motiva-tion of participation in physical activity. Journal of Sport Behavior, 27, 378390. 